\title{
Optical Properties of Manganese-Doped Individual CdTe Quantum Dots
}

\author{
L. Besombes ${ }^{a, *}$, Y. LÉGer ${ }^{a}$, L. Maingault ${ }^{a}$, D. Ferrand ${ }^{a}$, \\ C. Bougerol ${ }^{a}$, H. Mariette ${ }^{a}$ And J. Cibert $^{b}$ \\ ${ }^{a}$ CEA-CNRS group "Nanophysique et Semiconducteurs" \\ Laboratoire de Spectrométrie Physique, CNRS and Université J. Fourier \\ BP87, 38402 St Martin d'Heres, France \\ ${ }^{b}$ Laboratoire Louis Néel, CNRS, BP 166X, 38042 Grenoble, France
}

\begin{abstract}
The magnetic state of a single magnetic ion $\left(\mathrm{Mn}^{2+}\right)$ embedded in an individual quantum dot is optically probed using microspectroscopy. The fine structure of a confined exciton in the exchange field of a single $\mathrm{Mn}^{2+}$ ion $(S=5 / 2)$ is analyzed in detail. The exciton- $\mathrm{Mn}^{2+}$ exchange interaction shifts the energy of the exciton depending on the $\mathrm{Mn}^{2+}$ spin component and six emission lines are observed at zero magnetic field. The emission spectra of individual quantum dots containing a single magnetic $\mathrm{Mn}$ atom differ strongly from dot to dot. The differences are explained by the influence of the system geometry, specifically the in-plane asymmetry of the quantum dot and the position of the Mn atom. Depending on both these parameters, one has different characteristic emission features which either reveal or hide the spin state of the magnetic atom. The observed behavior in both zero field and under magnetic field can be explained quantitatively by the interplay between the exciton-manganese exchange interaction (dependent on the $\mathrm{Mn}$ position) and the anisotropic part of the electron-hole exchange interaction (related to the asymmetry of the quantum dot).
\end{abstract}

PACS numbers: 78.67.Hc, 78.55.Et, 75.75.+a

\section{Introduction}

Future devices for information storage, transport, and processing are likely to be based on individual quantum objects and to involve new physical properties. They could, for instance, combine manipulation of charges and manipulation of

${ }^{*}$ corresponding author; e-mail: lbesombes@spectro.ujf-grenoble.fr 
spins in what is now called spintronics. Semiconductor quantum dots (QDs) hold particular promise for these future devices. Such zero-dimensional structures have been proposed as building blocks for spin-based, solid state, quantum logic gates [1-3] in a fully scalable system. Developing such devices requires, however, the ability to detect and manipulate individual spins [4, 5]. Magnetic semiconductor quantum dots (QDs), where excitons (electron-hole pairs) can interact strongly with the magnetic atoms, hold particular promise as building blocks for such spin-based systems. In these low dimensional structures, the geometric factors that become more and more important with decreasing QD size (because of the quantum confinement) need to be considered with great care [6]. For instance, any in-plane asymmetry can introduce very strong effects in the case of small dots (energy shift of the quantum levels, induced linear polarization) as revealed by single dot optical spectroscopy $[7,8]$. Thus it is crucial, with nanometric scale magnetic objects, to understand and to control all the geometric parameters which characterize the system.

In a diluted magnetic semiconductor, the strong $s p-d$ exchange interactions between the band carriers and the transition metal ions give rise to large magneto-optical effects [9]. In a magnetic QD, this $s p-d$ interaction takes place with a single carrier or a single electron-hole pair. Recently, the formation of quasi zero-dimensional magnetic polarons (i.e. regions with correlated carrier and magnetic ion spins) has been demonstrated [10] in individual QDs. Up to now, however, all the experimental studies on diluted magnetic QDs were focused on the interaction of a single carrier spin with its paramagnetic environment (large number of magnetic atoms). Here we reveal the various spin states of a single magnetic ion interacting with a single electron-hole pair by investigating the magneto-optic properties of an individual self-assembled QD doped with a single $\mathrm{Mn}^{2+}$ ion.

In this article we show how we can optically probe the magnetic state of a single $\mathrm{Mn}^{2+}$ ion embedded in an individual QD. The fine structure of a confined exciton in the exchange field of a single $\mathrm{Mn}^{2+}$ ion is analyzed in detail. At zero magnetic field, the exchange coupling with the exciton lifts the degeneracy of the six $\mathrm{Mn}^{2+}$ spin components. We measure experimentally the parameters that will permit controlled manipulations of individual localized spins by single carriers. Particularly, we will show that the electron-hole-Mn system is very sensitive to the geometry, specifically the asymmetry of the QD and the position of the Mn atom in the dot. We report experimental results showing the three different types of spectra possible. Control of both the exciton-manganese (X-Mn) exchange interaction (determined by the position of the $\mathrm{Mn}$ ) and the anisotropic electronhole $(\mathrm{e}-\mathrm{h})$ exchange interaction (related to the shape of the dot) appears as a key condition for detection and manipulation of the spin state of the isolated magnetic atom. A strong interaction between the exciton and the Mn splits the six Mn spin components, but a strong anisotropy of the dot perturbs the spectrum pattern and can hide the information on the Mn spin state. 


\section{Samples and experiment}

In the magnetic QDs growth process, a low concentration of Mn is introduced into the QDs by adjusting the density of $\mathrm{Mn}$ atoms in the QD layer to be equal to the density of QDs $\left(\approx 5 \times 10^{9} \mathrm{~cm}^{-2}\right)$. The QDs are grown by molecular beam epitaxy. A $\mathrm{Zn}_{0.94} \mathrm{Mn}_{0.06}$ Te barrier followed by a 10 monolayer $\mathrm{ZnTe}$ spacer is deposited on a ZnTe substrate (Fig. 1b). The CdTe QD layer is then deposited and capped with a ZnTe barrier [11]. The Mn inter-mixing during the growth of the $\mathrm{ZnTe}$ spacer introduces a sparse distribution of $\mathrm{Mn}^{2+}$ ions in the QD layer [12].

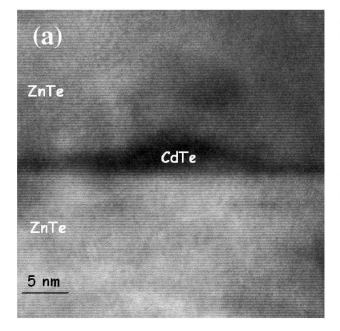

(b)

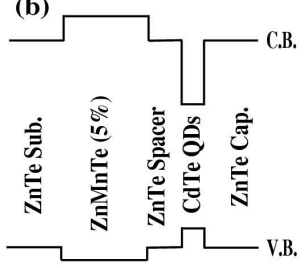

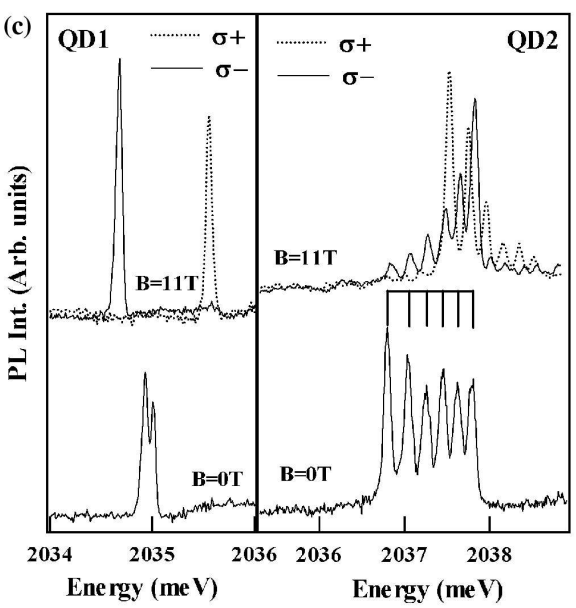

Fig. 1. (a) TEM image illustrating the self-assembled CdTe/ZnTe QDs used in this study. The QD density is about $5 \times 10^{9} \mathrm{~cm}^{-2}$. (b) Scheme of the sample structure. (c) Low temperature $(T=5 \mathrm{~K}) \mathrm{PL}$ spectra obtained at $B=0 \mathrm{~T}$ and $B=11 \mathrm{~T}$ for an individual non-magnetic CdTe/ZnTe QD (QD1) and one Mn-doped QDs (QD2).

The photoluminescence (PL) of individual QDs is excited with the $514 \mathrm{~nm}$ line of an argon laser and collected through a large numerical-aperture microscope objective and aluminum shadow masks with $0.2-1.0 \mu \mathrm{m}$ apertures. The PL is then dispersed by a 2 meter additive double monochromator and detected by a nitrogen cooled Si CCD.

\section{Exciton-manganese exchange interaction in symmetric quantum dots}

In Fig. 1c, PL spectra of an individual Mn-doped QD are compared to those of a non-magnetic $\mathrm{CdTe} / \mathrm{ZnTe}$ reference sample. In non-magnetic samples, narrow PL peaks (limited by the spectrometer resolution of $50 \mu \mathrm{eV}$ ) can be resolved, each attributed to the recombination of a single electron-hole pair in a single QD. Most 
of the individual emission peaks of magnetic single QDs are characterized by a rather large line width of about $0.5 \mathrm{meV}$. For some of these QDs, a fine structure can be resolved and six emission lines are clearly observed at zero magnetic field. The measured splitting changes from dot to dot. This fine structure splitting as well as the broadening is obviously related to the influence of the magnetic ions located within the spatial extent of the exciton wave function. The broadening previously observed in magnetic QDs has been attributed by Bacher et al. to the magnetic fluctuations of the spin projection of a large number of $\mathrm{Mn}^{2+}$ spins interacting with the confined exciton [10]. Here, in these low concentration Mn-doped samples, the observation of a fine structure shows that the QD exciton interacts with a single $\mathrm{Mn}^{2+}$ spin. In time-averaged experiments, the statistical fluctuations of a single $\mathrm{Mn}^{2+}$ ion $(S=5 / 2)$ can be described in terms of populations of its six spin states quantized along the direction normal to the QD plane. The exchange interaction of the confined exciton with the $\mathrm{Mn}^{2+}$ ion shifts its energy depending on the $\mathrm{Mn}^{2+}$ spin projection, resulting in the observation of six emission lines.

QDs with single $\mathrm{Mn}^{2+}$ ions were considered theoretically in the case of spherical nanocrystals with a strong confinement [13]. The eigenstates resulting from the exchange coupling of the exciton and the magnetic ion were obtained by a combination of the electron, hole, and $\mathrm{Mn}^{2+}$ magnetic moments. Here, in flat self-assembled QDs with a relatively weak confinement, the biaxial strains in the plane of the QD lift the degeneracy of the hole spin projections (heavy-hole/light-hole splitting). In a first approximation, our system can be described by a heavy-hole exciton confined in a symmetric QD in interaction with the six spin projections of the manganese ion. The spin interaction part of the Hamiltonian of this system is given by

$$
H_{\mathrm{int}}=I_{\mathrm{e}} \sigma \cdot S+I_{\mathrm{h}} j \cdot S+I_{\mathrm{eh}} \sigma \cdot j,
$$

where $I_{\mathrm{e}}\left(I_{\mathrm{h}}\right)$ is the Mn-electron (-hole) exchange integral, $I_{\mathrm{eh}}$ - the electron-hole exchange interaction and $\sigma(j)$ the magnetic moment of the electron (hole). The initial states of the transitions are obtained from the diagonalization of the spin Hamiltonian and Zeeman Hamiltonian in the subspace of the heavy hole exciton and $\mathrm{Mn}^{2+}$ spin components $| \pm 1 / 2\rangle_{\mathrm{e}}| \pm 3 / 2\rangle_{\mathrm{h}}\left|S_{z}\right\rangle_{\mathrm{Mn}}$, with $S_{z}= \pm 5 / 2, \pm 3 / 2, \pm 1 / 2$. Since the dipolar interaction operator does not affect the Mn $d$ electrons, the final states involve only the $\mathrm{Mn}^{2+}$ states $\left|S_{z}\right\rangle_{\mathrm{Mn}}$ with the same spin component [14].

In this framework, at zero magnetic field, the QD emission presents a fine structure composed of six doubly degenerate transitions roughly equally spaced in energy. The lower energy bright states, $|+1 / 2\rangle_{\mathrm{e}}|-3 / 2\rangle_{\mathrm{h}}|+5 / 2\rangle_{\mathrm{Mn}}$ and $|-1 / 2\rangle_{\mathrm{e}}$ $|+3 / 2\rangle_{\mathrm{h}}|-5 / 2\rangle_{\mathrm{Mn}}$ are characterized by an antiferromagnetic coupling between the hole and the $\mathrm{Mn}^{2+}$ ion. The following states are associated with the $\mathrm{Mn}^{2+}$ spin projections $S_{z}= \pm 3 / 2, \pm 1 / 2$ until the higher energy states $|-1 / 2\rangle_{\mathrm{e}}|+3 / 2\rangle_{\mathrm{h}}$ $|+5 / 2\rangle_{\mathrm{Mn}}$ and $|+1 / 2\rangle_{\mathrm{e}}|-3 / 2\rangle_{\mathrm{h}}|-5 / 2\rangle_{\mathrm{Mn}}$ corresponding to ferromagnetically coupled hole and manganese. In this simple model the zero field splitting 
$\delta_{\mathrm{Mn}}=\frac{1}{2}\left(I_{\mathrm{e}}-3 I_{\mathrm{h}}\right)$ depends only on the exchange integrals $I_{\mathrm{e}}$ and $I_{\mathrm{h}}$ and is thus related to the position of the $\mathrm{Mn}^{2+}$ ion within the exciton wave function.

\section{Interplay between electron-hole and exciton-manganese exchange interaction}

In single Mn-doped QDs, a deviation from the predictions of the simple model presented previously is often observed. Figure 2 shows three types of emission spectra obtained at $5 \mathrm{~K}$, under low excitation density, for single QDs containing a single Mn. In the first example (QD3), a structure composed of six main lines dominates the emission spectrum at zero magnetic field. These lines correspond to the radiative ("bright") exciton states $J_{z}= \pm 1$ coupled to the six spin components of the Mn atom $(S=5 / 2)$. Analysis of the line intensities gives the occupation probability of the six Mn spin states [14]. The three low intensity lines on the low energy side of the structure (Fig. 1a) can be attributed to the contribution of exciton dark states [15]. We will see that a fine structure with well separated lines requires not only that the Mn atom interacts strongly with the exciton in the dot, but also that the dot must retain high symmetry.

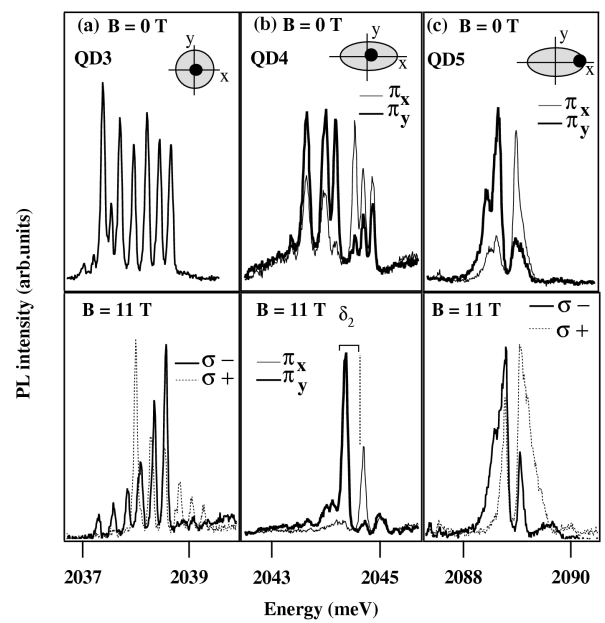

Fig. 2. Low temperature $(T=5 \mathrm{~K}) \mathrm{PL}$ spectra of three different Mn-doped CdTe/ZnTe QDs at $B=0 \mathrm{~T}$ and $B=11 \mathrm{~T} . \sigma^{ \pm}$are circular polarizations and $\pi_{x, y}$ are two orthogonal linear polarizations. (a) Exciton strongly coupled with a $\mathrm{Mn}$ atom, in a symmetric dot (QD3). (b) Exciton strongly coupled with a Mn atom, in an asymmetric dot (QD4). (c) Exciton less strongly coupled with a Mn atom, in an asymmetric dot (QD5).

By contrast, the emission of QD5 consists of two broad peaks with width about $200 \mu \mathrm{eV}$, separated by an energy gap of about $300 \mu \mathrm{eV}$. A similar gap is seen clearly in the third example (QD4), for which six lines are observed, but with two 
sets of three lines separated by the central gap. An additional essential property is that the emission lines are linearly polarized along two orthogonal directions for QD4 and QD5, whereas for QD2 and QD3 the emission is unpolarized.

Such large differences in the zero field emission spectra can be attributed to competition between the $\mathrm{X}-\mathrm{Mn}$ interaction and the anisotropic $\mathrm{e}-\mathrm{h}$ exchange interaction arising in asymmetric QDs. We recall that for non-magnetic QDs, the $\mathrm{e}-\mathrm{h}$ exchange interaction in an anisotropic potential mixes the bright exciton states $J_{z}= \pm 1$. The emission of the QD is then linearly polarized along two orthogonal directions and split by the anisotropic exchange energy $\delta_{2}[7,16]$, originating from the long range (non-analytic) e-h exchange interaction $H_{\mathrm{e}-\mathrm{h}}^{\text {aniso }}$ [17].

Let us now consider how the simultaneous presence of $H_{\mathrm{e}-\mathrm{h}}^{\text {anis }}$ and the $\mathrm{X}-\mathrm{Mn}$ exchange interaction affects the zero field spectrum. Diagonalizing the augmented Hamiltonian $H_{\text {int }}+H_{\mathrm{e}-\mathrm{h}}^{\text {anis }}$ shows that the e-h exchange interaction splits the six line structure into two subsets of three lines. Figure 3 represents the bright state transitions $\left(J_{z}= \pm 1\right)$ associated with the six manganese spin projections $S_{z}$. The anisotropic $\mathrm{e}-\mathrm{h}$ exchange term $\delta_{2}$ mixes the bright exciton states associated with the same Mn spin projection, inducing an extra splitting between them. The energy splitting of the bright excitons for a given value of $S_{z}$ is given by

$$
\Delta E\left(S_{z}\right)=\sqrt{\delta_{2}^{2}+\left(2\left|S_{z}\right| \delta_{\mathrm{Mn}}\right)^{2}}
$$

where $2\left|S_{z}\right| \delta_{\mathrm{Mn}}$ is the splitting induced by the Mn only. The mixing induced by $\delta_{2}$ is thus strongest for the central pair of states, associated with the $S_{z}= \pm 1 / 2$ Mn spin projections. Equation (2) for $\Delta E\left(S_{z}\right)$ shows that anisotropy of the dot creates a gap in the center of the emission structure, see Fig. 2. This explains the line patterns observed for the three types of $\mathrm{QD}$ spectra presented above.

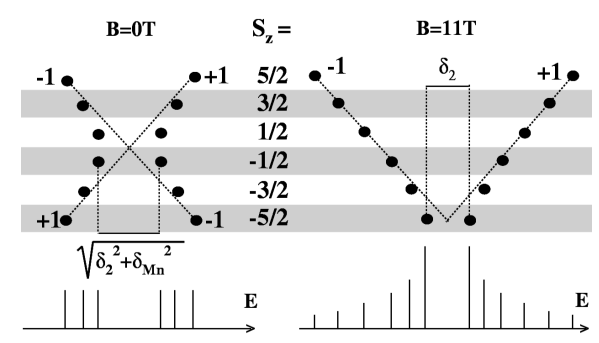

Fig. 3. Bright state transitions in an asymmetric Mn-doped QD at $B=0 \mathrm{~T}$ and $11 \mathrm{~T}$. The $\mathrm{e}-\mathrm{h}$ exchange interaction induces anticrossings (energy $\delta_{2}$ ) of the \pm 1 excitons associated with $S_{z}=-1 / 2,-3 / 2,-5 / 2$ for successive values of $B$; the $S_{z}=-5 / 2$ anticrossing is shown at right.

The width of the central gap $\left(\sqrt{\delta_{2}^{2}+\delta_{\mathrm{Mn}}^{2}}\right)$ and the width of the whole six-line spectrum structure $\left(\sqrt{\delta_{2}^{2}+\left(5 \delta_{\mathrm{Mn}}\right)^{2}}\right)$ yield the values of $\delta_{2}$ and $\delta_{\mathrm{Mn}}$ for the three dots of Fig. 2. The ratio $\delta_{2} / \delta_{\mathrm{Mn}}$ determines the spectrum type. For QD3, the ratio $\delta_{2} / \delta_{\mathrm{Mn}}$ is found to be $<0.2$, with $\delta_{\mathrm{Mn}}=250 \mu \mathrm{eV}$ : the $\mathrm{X}-\mathrm{Mn}$ interaction 
dominates the emission structure and the Mn spin states are directly resolved in the optical spectrum. By contrast, $\delta_{2} / \delta_{\mathrm{Mn}}=2.3$ for QD5 $\left(\delta_{\mathrm{Mn}}=120 \mu \mathrm{eV}\right)$, so the anisotropy splitting predominates: we observe only two broad peaks separated by a central gap and can no longer resolve the Mn spin states. A reduction in $\delta_{\mathrm{Mn}}$ of only a factor of two has completely changed the type of spectrum. QD4 demonstrates the intermediate case $\left(\delta_{2} / \delta_{\mathrm{Mn}}=1.3\right.$ with $\left.\delta_{\mathrm{Mn}}=230 \mu \mathrm{eV}\right)$ where the combined effect of the $\mathrm{e}-\mathrm{h}$ and $\mathrm{X}-\mathrm{Mn}$ exchange interactions is seen very clearly: despite the importance of anisotropy, the Mn spin states are still resolved as two subsets of three lines separated by the central gap.

The parameter $\delta_{\mathrm{Mn}}$ is determined by the values of $I_{\mathrm{e}}=\alpha\left|\phi_{\mathrm{e}}\left(R_{\mathrm{Mn}}\right)\right|^{2}$ and $I_{\mathrm{h}}=(\beta / 3)\left|\phi_{\mathrm{h}}\left(R_{\mathrm{Mn}}\right)\right|^{2}[18]$. Here $\phi_{\mathrm{e}}\left(\phi_{\mathrm{h}}\right)$ is the electron (hole) envelope function, which falls off with $R_{\mathrm{Mn}}$, the distance of the Mn atom from the QD center; $\alpha(\beta)$ are the $\mathrm{Mn}-\mathrm{e}(\mathrm{h})$ exchange constants [9]. Clearly, for QD5, the Mn atom is further from the dot center than for QD2, QD3, and QD4.

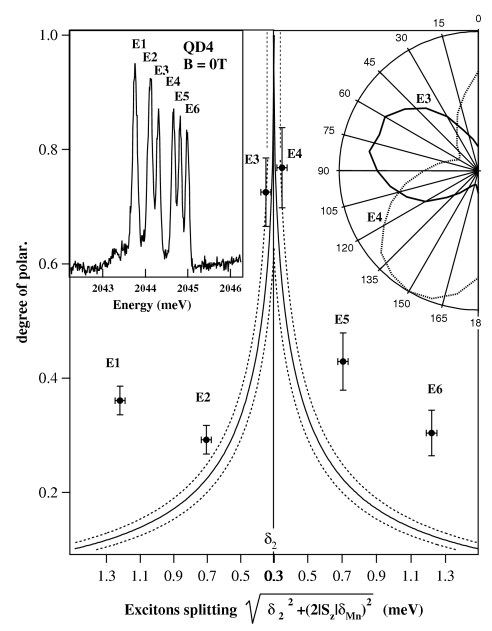

Fig. 4. Measured degree of linear polarization of emission lines E1-E6 for QD4 in zero field, as a function of the splitting between the $\left|J_{z}= \pm 1, S_{z}\right\rangle$ states, compared to theoretical behavior (full curves; dotted curves show the uncertainty range corresponding to the imprecision in $\delta_{2}$ ). The left inset labels the emission lines. The right inset shows a polar plot of the PL intensity of the lines E3 and E4 $\left(S_{z}= \pm 1 / 2\right)$ as a function of the linear polarizer orientation.

The theory also explains the linear polarization that we observe in zero magnetic field. When anisotropic electron-hole exchange interaction is included, the eigenstates of the $\mathrm{X}-\mathrm{Mn}$ system are of the form $[8,16]$ :

$$
\begin{aligned}
& |+\rangle\left|S_{z}\right\rangle=(\cos \theta|+1\rangle+\sin \theta|-1\rangle)\left|S_{z}\right\rangle, \\
& |-\rangle\left|S_{z}\right\rangle=(\cos \theta|-1\rangle-\sin \theta|+1\rangle)\left|S_{z}\right\rangle,
\end{aligned}
$$


where $\tan 2 \theta=\delta_{2} /\left(2\left|S_{z}\right| \delta_{\mathrm{Mn}}\right)$. The mixing of the bright states associated with a Mn spin state $S_{z}$ is controlled by the ratio of $\delta_{2}$ to the Mn induced splitting $2\left|S_{z}\right| \delta_{\mathrm{Mn}}$. The emission lines have partial linear polarization along two orthogonal directions corresponding to the principal axes of the anisotropic potential. Figure 4 compares measured and theoretical degree of polarization for QD4. The data are in qualitative agreement with the theoretical curve. In particular, central lines E3 and E4, associated with $S_{z}= \pm 1 / 2$, are almost completely polarized. The expected decrease with increase in $\Delta E\left(S_{z}\right)$ is reproduced qualitatively especially for the three upper energy lines E4, E5, and E6. For the lower energy lines, the degree of polarization could be influenced by the non-radiative states $\left(J_{z}= \pm 2\right)$ which lie in their energy range.

\section{Spin properties of manganese-doped quantum dots under magnetic field}

When an external magnetic field is applied in the Faraday geometry (Fig. 5a), each PL peak is further split and twelve lines are observed, six in each circular polarization. The Zeeman effect of the Mn states is identical in the initial and final state of the optical transitions and the six lines in a given polarization follow the Zeeman and diamagnetic shift of the exciton, as in a non-magnetic QD. The parallel evolution of six lines is perturbed around $7 \mathrm{~T}$ in $\sigma^{-}$polarization by anticrossings observed for five of the lines. In addition, as the magnetic field increases, one line in each circular polarization increases in intensity and progressively dominates the spectrum.

The electron- $\mathrm{Mn}^{2+}$ part of the interaction Hamiltonian $I_{\mathrm{e}}(\sigma \cdot S)$ couples the dark $\left(J_{z}= \pm 2\right)$ and bright $\left(J_{z}= \pm 1\right)$ heavy hole exciton states. This coupling corresponds to a simultaneous electron and $\mathrm{Mn}^{2+}$ spin flip changing a bright exciton into a dark exciton. Because of the strain induced splitting of light-hole and heavy-hole levels, a similar $\mathrm{Mn}^{2+}$-hole spin flip scattering is not allowed. The electron- $\mathrm{Mn}^{2+}$ spin flip is enhanced as the corresponding levels of bright and dark excitons are brought into coincidence by the Zeeman effect. An anticrossing is observed around $7 \mathrm{~T}$ for five of the bright states in $\sigma^{-}$polarization (experiment: Fig. 5a and theory: Fig. 5b). It induces a transfer of oscillator strength to the dark states. In agreement with the experimental results, in the calculations the lower energy state in $\sigma^{-}$polarization $\left(|+1 / 2\rangle_{\mathrm{e}}|-3 / 2\rangle_{\mathrm{h}}|+5 / 2\rangle_{\mathrm{Mn}}\right)$ does not present any anticrossing. In this spin configuration, both the electron and the $\mathrm{Mn}^{2+}$ ion have maximum spin projection and a spin flip is not possible.

The minimum energy splitting at the anticrossing is directly related to the electron- $\mathrm{Mn}^{2+}$ exchange integral $I_{\mathrm{e}}$. For instance, the splitting measured for the higher energy line in $\sigma^{-}$polarization (Fig. $2 \mathrm{c}$ ), $\Delta E=150 \mu \mathrm{eV}$ gives $I_{\mathrm{e}} \approx 70 \mu \mathrm{eV}$. From the overall splitting measured at zero field $(1.3 \mathrm{meV})$ and with this value of $I_{\mathrm{e}}$, we obtain $I_{\mathrm{h}} \approx-150 \mu \mathrm{eV}$. These values are in good agreement with values 

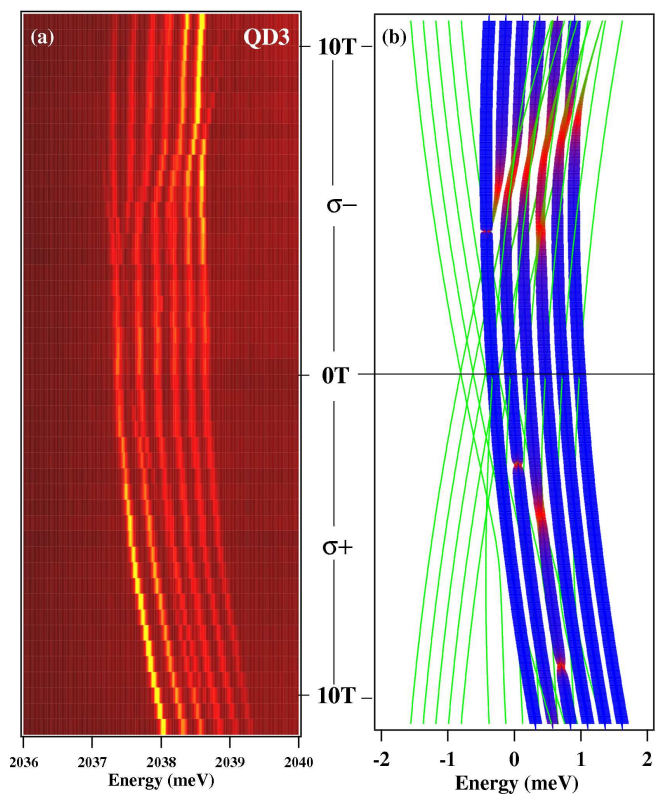

Fig. 5. (a) Magnetic field dependence of the emission of a symmetric Mn-doped QD (QD3) showing the anticrossing of bright and dark states around 6 T. (b) Optical transitions obtained from the diagonalization of the spin + Zeeman + diamagnetism Hamiltonian in the subspace of the 24 heavy-hole exciton and Mn spin components. Line thickness and color scale for $\sigma^{+}, \sigma^{-}$are proportional to absolute value of the projection of the exciton state on the $J_{z}=+1,-1$ exciton, respectively (green $=$ low intensity, blue $=$ high intensity). The two transitions which are forbidden at all magnetic fields $\left(\left|J_{z}= \pm 2, S_{z}=\mp 5 / 2\right\rangle\right)$ are not plotted. The calculation is performed with $g_{\mathrm{e}}=-0.9$, $g_{\mathrm{h}}=0.45, g_{\mathrm{Mn}}=2$ and $I_{\mathrm{eh}}=0.66 \mathrm{meV}$ (corresponding to a singlet-triplet splitting $\left.\hbar \omega_{\mathrm{ST}}=0.7 \mathrm{meV}\right), I_{\mathrm{e}}=0.07 \mathrm{meV}, I_{\mathrm{h}}=-0.15 \mathrm{meV}$ and $\gamma=2.45 \mu \mathrm{eV} \mathrm{T} \mathrm{T}^{-2}$.

estimated from a modeling of the QD confinement by a square quantum well in the growth direction and a truncated parabolic potential in the QD plane. With a quantum well thickness $L_{z}=3 \mathrm{~nm}$ and a Gaussian wave function characterized by an in-plane localization parameter $\xi=5 \mathrm{~nm}$ we obtain $I_{\mathrm{e}} \approx 65 \mu \mathrm{eV}$ for a $\mathrm{Mn}^{2+}$ ion placed at the center of the QD.

However, the ratio of the exchange integral $\left(3 I_{\mathrm{h}}\right) / I_{\mathrm{e}} \approx-6$ for QD3 does not directly reflect the ratio of the $s p-d$ exchange constants $\beta / \alpha \approx-4$ measured in bulk CdMnTe alloys [9]. This deviation likely comes from the difference in the electron-Mn and hole-Mn overlap expected from the difference in the electron and hole confinement length but could also be due to a change of the exchange parameters induced by the confinement [19]. A dispersion of the zero field energy splitting observed from dot to dot is then due to a variation of the Mn-exciton overlap for different QDs. However, this model does not reproduce the observed 
non-uniform zero field splitting (Fig. 1c). A more accurate model should take into account the full valence band structure and the heavy-hole light-hole mixing.

As illustrated in Fig. 5, the relative intensities of the six emission lines observed in each circular polarization depends strongly on the applied magnetic field. The emission intensity, which is almost equally distributed over the six emission lines at zero field, is concentrated on the high energy line of the $\sigma^{-}$emission and on the low energy line of the $\sigma^{+}$emission at high magnetic field. As the magnetic field increases, the $\mathrm{Mn}^{2+}$ ion is progressively polarized. In time-averaged experiments, the probability to observe the recombination of the bright excitons coupled with the $S_{z}=-5 / 2$ spin projection is then enhanced. Two states dominate the spectra: $|-1 / 2\rangle_{\mathrm{e}}|+3 / 2\rangle_{\mathrm{h}}|-5 / 2\rangle_{\mathrm{Mn}}$ in the low energy side of the $\sigma^{+}$emission and $|+1 / 2\rangle_{\mathrm{e}}|-3 / 2\rangle_{\mathrm{h}}|-5 / 2\rangle_{\mathrm{Mn}}$ in the high energy side of the $\sigma^{-}$polarization. Changing the temperature of the $\mathrm{Mn}^{2+}$ ion will affect the distribution of the exciton emission intensities. The PL of the exciton is then a direct probe of the magnetic state of the $\mathrm{Mn}^{2+}$ ion.

In anisotropic QDs, the interplay between the electron-hole and excitonMn exchange interactions is confirmed by magneto-optical measurements (Figs. 5 and 6). For QD3 (Fig. 5a) and QD4 (Fig. 6a), the typical Zeeman splitting of the six lines is clearly observed in the data at all fields, with a strong intensity gradient at the highest fields (see Fig. 2a) resulting from a rather strong Mn spin polarization. For the clearly anisotropic dots (QD4, Fig. 6), the central gap in the emission structure is maintained in both circular polarizations, with a small quadratic diamagnetic energy shift. This behavior is explained as follows. The dot anisotropy leads to successive anticrossings of the \pm 1 exciton states associated with given Mn spin projections $(-1 / 2,-3 / 2$, and $-5 / 2)$ as a function of magnetic field. As $B$ increases, transitions associated with the $J_{z}=+1$ exciton shift up in energy whereas the $J_{z}=-1$ transitions shift down (see Fig. 3). The anisotropic part of the electron-hole exchange interaction mixes successively the $J_{z}= \pm 1$ exciton states associated with $S_{z}=-1 / 2$, then with $S_{z}=-3 / 2$ and finally with $S_{z}=-5 / 2$ at successively higher $B$. For QD3, these anticrossings are observed successively at $2.5,7$, and $11 \mathrm{~T}$.

To understand fully the rich magnetic behavior of dots like QD4, we calculated the optical transitions under magnetic field by diagonalizing the complete Hamiltonian of the electron-heavy hole-Mn system (including the exchange, Zeeman, and diamagnetism Hamiltonians). Calculated transitions are presented in Fig. 6b. The fitted Landé factors of the electron $\left(g_{\mathrm{e}}=-1.1\right)$, the hole $\left(g_{\mathrm{h}}=0.3\right)$ and the $\mathrm{Mn}$ atom $\left(g_{\mathrm{Mn}}=2.0\right)$, the splitting between $J_{z}= \pm 1$ and $J_{z}= \pm 2$ excitons $(=1 \mathrm{meV})$ and the diamagnetic factor $\left(\gamma=2.45 \mu \mathrm{eV} \mathrm{T} \mathrm{T}^{-2}\right)$ agree well with previous works $[8,14]$. Parameters $\delta_{2}$ and $\delta_{\mathrm{Mn}}$ were adjusted to fit the zero field data, as explained earlier.

Comparison between calculation and data explains most of the details of the magneto-optic properties of QD4. In particular, around $7 \mathrm{~T}$, the central gap is 


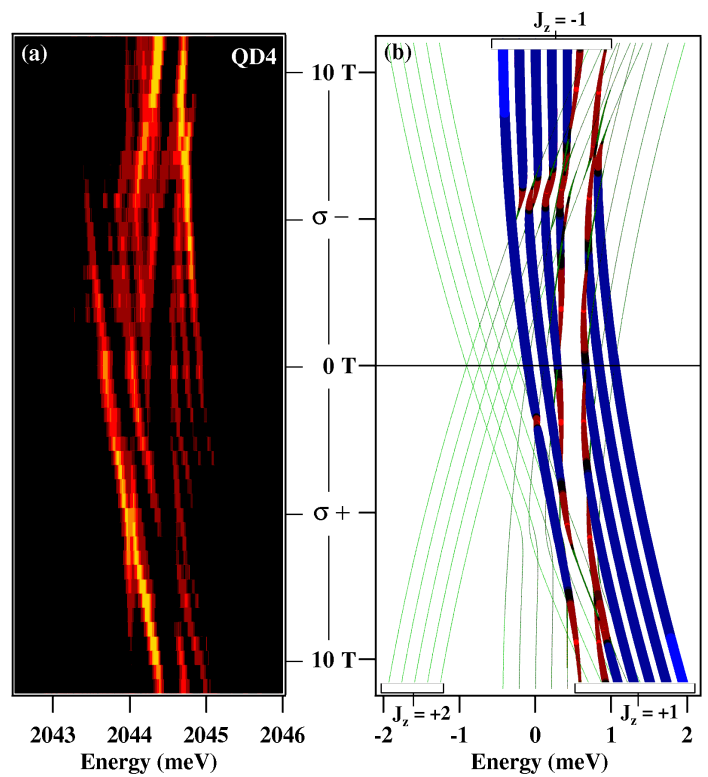

Fig. 6. (a) Intensity map of magnetic field dependence of the emission spectrum of asymmetric Mn-doped dot QD4, for circular polarization $\sigma^{+}$and $\sigma^{-}$. (b) Optical transitions obtained from the diagonalization of the spin+Zeeman+diamagnetism Hamiltonian in the subspace of the 24 heavy-hole exciton and Mn spin components. Line thickness and color scale for $\sigma^{+}, \sigma^{-}$are proportional to absolute value of the projection of the exciton state on the $J_{z}=+1,-1$ exciton respectively (green $=$ low intensity, blue $=$ high intensity). The two transitions which are forbidden at all magnetic fields $\left(\left|J_{z}= \pm 2, S_{z}=\mp 5 / 2\right\rangle\right)$ are not plotted. See the text for the parameters used in the calculation.

perturbed in both circular polarizations. In $\sigma^{-}$, this is due to anticrossings induced by the mixing of $\left|s_{\mathrm{e} z}=1 / 2, j_{\mathrm{h} z}=-3 / 2, S_{z}\right\rangle$ states and $\left|-1 / 2,-3 / 2, S_{z}+1\right\rangle$ states by the electron-Mn exchange [14], i.e. corresponding to simultaneous spin-flips of electron and manganese spins. In $\sigma^{+}$polarization, Fig. $6 \mathrm{~b}$ shows that the line of second lowest energy crosses the central gap as an essentially non-radiative transition. This implies a mixing of $|-1 / 2,3 / 2,-3 / 2\rangle$ and $|-1 / 2,-3 / 2,-1 / 2\rangle$. This is a second order mixing involving both mixing of $|-1 / 2,-3 / 2,-1 / 2\rangle$ and $|1 / 2,-3 / 2,-3 / 2\rangle$ by the e-Mn exchange and mixing of $|-1 / 2,3 / 2,-3 / 2\rangle$ and $|1 / 2,-3 / 2,-3 / 2\rangle$ by the anisotropic e- $\mathrm{h}$ exchange; that is, the e-Mn exchange induces a mixing of states mediated by the anisotropy-induced coupling.

\section{Quantum dots doped with several manganese atoms}

Finally, let us note that in these low Mn density QD samples, most of the experimentally observed PL spectra lack of a simple interpretation in terms of a 
well defined spin system and look closer to the PL spectrum of QD7 (Fig. 7). QD7 shows a zero field broad emission line $(\mathrm{FWHM} \approx 1 \mathrm{meV})$ that narrows upon application of a magnetic field in the Faraday configuration. This emission spectrum definitely corresponds to a single quantum dot coupled with a few Mn atoms. For

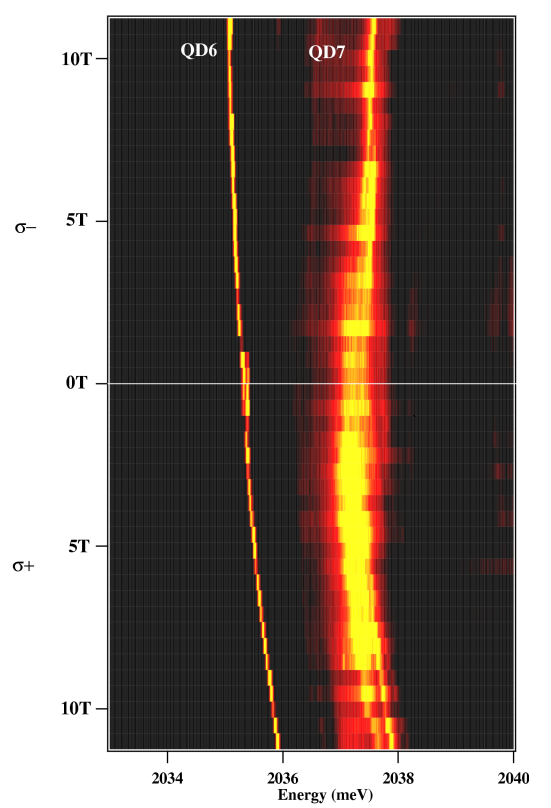

Fig. 7. Intensity map of magnetic field dependence of the low temperature $(5 \mathrm{~K})$ emission spectrum of a single quantum dot doped with several Mn atoms (QD7), for circular polarization $\sigma^{+}$and $\sigma^{-}$, compared with the emission of an undoped quantum dot (QD6).

more than one $\mathrm{Mn}$, the resulting emission fine structure can no longer be resolved. The large line width emission is related to statistical magnetic fluctuations: statistical variations of the magnetization within the exciton wave function result in a broadening of the single QD emission in time integrated experiment. The line width narrowing in magnetic field is connected to the suppression of statistical fluctuations due to the alignment of the Mn spins [20]. This alignment of the Mn spins is also responsible of the observed initial red-shift and polarization of the emission observed at low field (below $8 \mathrm{~T}$ ) and can be related to the classical giant Zeeman effect in diluted magnetic semiconductors. At higher field (above $10 \mathrm{~T}$ ) the Zeeman splitting of the exciton compensate the splitting induced by the exchange interaction with Mn showing that QD7 is indeed coupled with a very low number of magnetic atoms. As the QDs' PL spectra are most sensitive to the Mn spins that are strongly coupled to the exciton, even when many Mn atoms are present in the QD, the PL might be probing only those with a strong overlap with the confined carriers. 


\section{Conclusion}

In summary, we have shown that the emission of a QD could be used to probe the spin state of an individual magnetic atom. We demonstrated that the position of the $\mathrm{Mn}$ atom in the $\mathrm{QD}$ is not the only parameter that has to be controlled in order to resolve the Mn spin states. Another geometric parameter must be considered: the asymmetry of the dot. The interplay between these two parameters has important consequences for the QD optical properties. The Mnexciton exchange interaction tends to separate the bright exciton states, whereas the anisotropic part of the electron-hole exchange interaction tends to couple them and to hide the Mn spin splitting. The wealth of data obtained gives a unified picture of the effects of dot asymmetry on the fine structure and polarization properties of optical transitions in single Mn-doped QDs. This allows us to determine the conditions required to tune the magnetic QD states in order to control and manipulate individual localized spins by single carriers. More generally, the interaction between the magnetic ion and the carriers (or exciton) in the QD could be exploited to manipulate the quantum state of an individual spin by optical or electrical injection of polarized carriers. Coherent manipulation of the spin state of a single magnetic ion could also be performed under pulsed resonant optical excitation, suggesting implementation of controlled spin-qubit operations.

\section{Acknowledgments}

The authors are grateful to Y. Genuist and M. Falco for expert technical assistance. This work was supported in part by CNRS ACI-DECORESS.

\section{References}

[1] D. Loss, D.P. DiVincenzo, Phys. Rev. A 57, 120 (1998).

[2] D.P. DiVincenzo, D. Bacon, J. Kempe, G. Burkard, K.B. Whaley, Nature 408, 339 (2000).

[3] M.N. Leuenberger, D. Loss, Nature 410, 789 (2001).

[4] B.E. Kane, Nature 393, 133 (1998).

[5] A.L. Efros, E.I. Rashba, M. Rosen, Phys. Rev. Lett. 87, 206601 (2001).

[6] M. Bayer, A. Kuther, A. Forchel, A. Gorbunov, V.B. Timofeev, F. Schäfer, J.P. Reithmaier, T.L. Reinecke, S.N. Walck, Phys. Rev. Lett. 82, 1748 (1999).

[7] V.D. Kulakovskii, G. Bacher, R. Weigand, T. Kümmell, A. Forchel, E. Borovitskaya, K. Leonardi, D. Hommel, Phys. Rev. Lett. 82, 1780 (1999).

[8] L. Besombes, K. Kheng, D. Martrou, Phys. Rev. Lett. 85, 425 (2000); L. Besombes, L. Marsal, K. Kheng, T. Charvolin, Le Si Dang, A. Wasiela, H. Mariette, J. Cryst. Growth 214, 742 (2000).

[9] J.K. Furdyna, J. Appl. Phys. 64, R29 (1988). 
[10] G. Bacher, A.A. Maksimov, H. Schömig, V.D. Kulakovskii, M.K. Welsch, A. Forchel, P.S. Dorozhkin, A.V. Chernenko, S. Lee, M. Dobrowolska, J.K. Furdyna, Phys. Rev. Lett. 89, 127201 (2002).

[11] F. Tinjod, B. Gilles, S. Moehl, K. Kheng, H. Mariette, Appl. Phys. Lett. 82, 4340 (2003).

[12] W. Grieshaber, A. Haury, J. Cibert, Y. Merle d'Aubigné, A. Wasiela, J.A. Gaj, Phys. Rev. B 53, 4891 (1996).

[13] A.K. Bhattacharjee, J. Pérez-Conde, Phys. Rev. B 68, 045303 (2003).

[14] L. Besombes, Y. Léger, L. Maingault, D. Ferrand, H. Mariette, J. Cibert, Phys. Rev. Lett. 93, 207403 (2004).

[15] L. Besombes, K. Kheng, L. Marsal, H. Mariette, Phys. Rev. B 65, 121314 (2002).

[16] E.L. Ivchenko, Phys. Status Solidi A 164, 487 (1997).

[17] G.E. Pikus, G.L. Bir, Sov. Phys. JETP 33, 108 (1971).

[18] F.V. Kyrychenko, J. Kossut, Phys. Rev. B 70, 205317 (2004).

[19] I.A. Merkulov, D.R. Yakovlev, A. Keller, W. Ossau, J. Geurts, A. Waag, G. Landwehr, G. Karczewski, T. Wojtowicz, J. Kossut, Phys. Rev. Lett. 83, 1431 (1999).

[20] P.S. Dorozhkin, A.V. Chernenko, V.D. Kulakovskii, A.S. Brichkin, A.A. Maksimov, H. Schoemig, G. Bacher, A. Forchel, S. Lee, M. Dobrowolska, J.K. Furdyna, Phys. Rev. B 68, 195313 (2003). 